\title{
Le oscillazioni libere del golfo di Civitavecchia e l'azione della viscosità cinematica
}

\author{
P. Caloi - M. C. Spadea
}

I. - E nostro proposito di studiare le oscillazioni libere caratteristiche dei principali golfi italiani, proseguendo la ricerca già iniziata con i golfi di Trieste $\left(^{(}\right)$e di Napoli $\left(^{2}\right)$.

Oggetto del presente lavoro è lo studio delle oscillazioni libere del golfo di Civitavecchia.

$\mathrm{E}$ ben noto che tale studio presuppone la conoscenza della batimetria del bacino in esame, che consenta il calcolo di elementi idrografici indispensabili all'applicazione dei vari metodi idrodinamici. Come base del rilevamento degli elementi sopra accennati è stata scelta la carta dell'Istituto Idrografico della Marina, scala $1: 5000$. Il golfo è stato suddiviso in 19 sezioni trasversali distanti 50 metri l'una dall'altra. Le aree di dette sezioni e dei corrispondenti elementi di superficie fra sezione e sezione sono riportati nella Tabella 1.

E stato da noi applicato il metodo di Goldberg, la teoria del quale è stata già esposta in precedenti lavori ( $\left.{ }^{3}\right)$. I calcoli sono stati piuttosto laboriosi poichè, contrariamente alle precedenti applicazioni, i risultati ottenuti erano sempre troppo discosti dai dati di osservazione.

Abbiamo dovuto limitarci ad un valore approssimato per difetto, quale risulta dalla Tabella 1 che, anche dopo la correzione di bocea, ha dato per il periodo dell'uninodale un valore di $6^{\text {m }}$ circa. Tale valore è evidentemente troppo discosto da quello fornito dall'osservazione, per le oscillazioni libere dell'uninodale, pari a circa $10^{\mathrm{m}}, 8$. Forse a causa della complessità del golfo e della sua limitata profondità, questa volta il metodo di Goldberg non poteva dare valori più approssimati.

Ad ogni modo, essi sono risultati troppo bassi per poterne attribuire la causa soltanto ai dati morfometrici.

Va qui notato che il valore di maggiore approssimazione lo ha fornito questa volta la formula di Merian, applicata sulla base di una lun- 


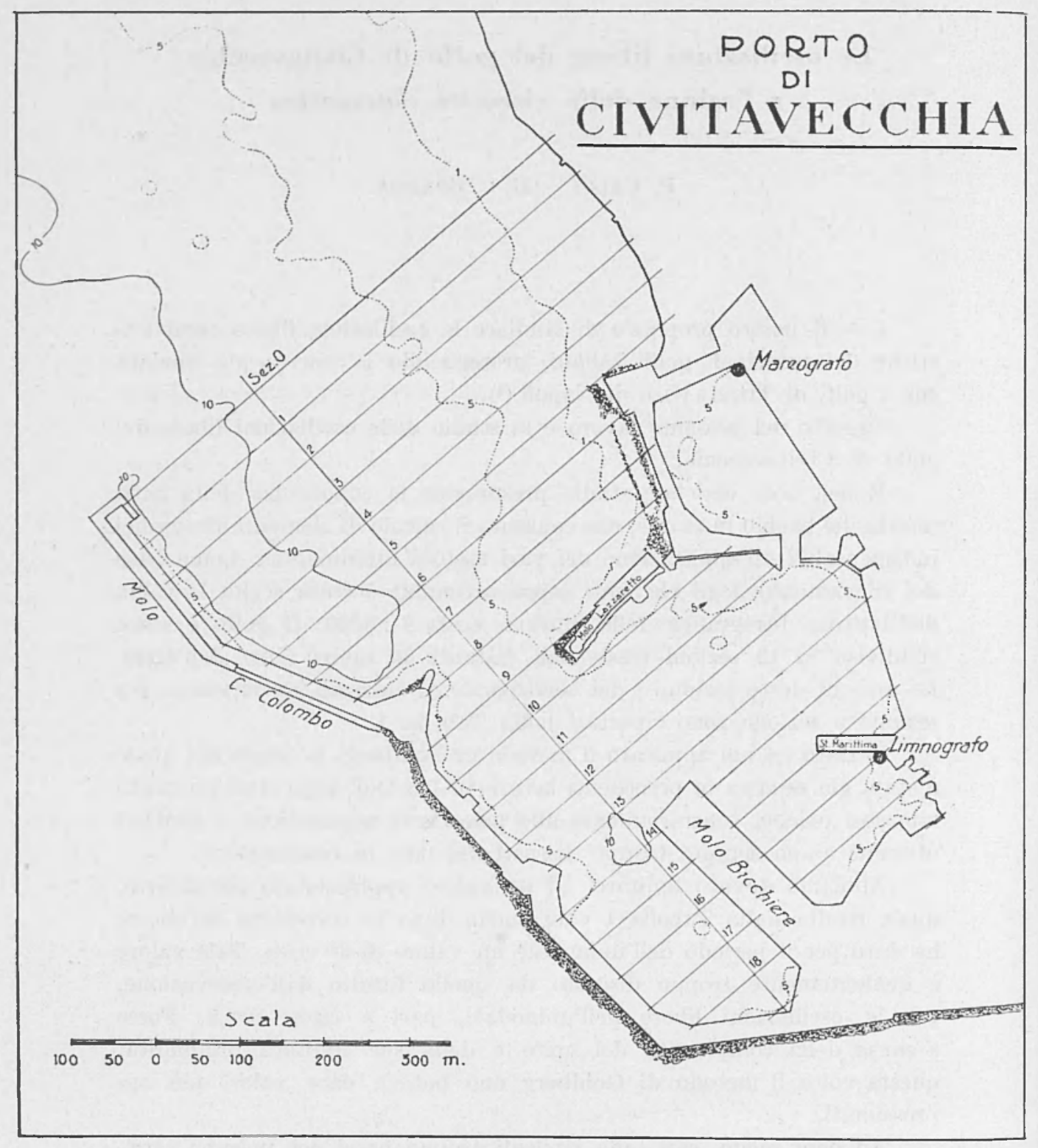

Fig. 1 
Taholla 1

\begin{tabular}{|c|c|c|c|c|c|c|}
\hline \multirow[b]{2}{*}{ Sezioni } & \multirow{2}{*}{$\begin{array}{c}A(x) \\
\mathrm{m}\end{array}$} & \multirow{2}{*}{$\begin{array}{c}S(x) \\
10^{2} \mathrm{~m}^{2}\end{array}$} & \multirow{2}{*}{$\begin{array}{l}\Delta v(x) \\
10^{2} \mathrm{~m}^{2}\end{array}$} & \multicolumn{3}{|c|}{$T:=5^{\mathrm{m}, 2}=312^{\mathrm{s}}$} \\
\hline & & & & $\begin{array}{c}\xi(x) \\
m\end{array}$ & $\begin{array}{c}\eta(x) \\
\mathrm{m}\end{array}$ & $\underset{5.10^{6} \mathrm{~m}^{3}}{\mathrm{~m}}$ \\
\hline 0 & 0 & 42.50 & 0 & 235.3 & 0 & 1.000 \\
\hline 1 & 50 & 35.00 & 328.75 & 283.4 & 0.4882 & 0.992 \\
\hline 2 & 50 & 36.75 & 332.50 & 265.0 & 1.0762 & 0.974 \\
\hline 3 & 50 & 40.75 & 332.50 & 232.4 & 1. 6260 & 0.947 \\
\hline 4 & 50 & 39.00 & 330.00 & 233.8 & 2.1082 & 0.912 \\
\hline 5 & 50 & 37.75 & 313.75 & 230.7 & 2.5933 & 0.871 \\
\hline 6 & 50 & 28.25 & 263.75 & 293.8 & 3.0720 & 0.830 \\
\hline 7 & 50 & 25.75 & 262.00 & 303.7 & 3.6816 & 0.782 \\
\hline 8 & 50 & 20.65 & 267.50 & 350.6 & 4.3118 & 0.724 \\
\hline 9 & 50 & 20.40 & 248.75 & 324.0 & 5.0393 & 0.661 \\
\hline 10 & 50 & 26.80 & 203.75 & 225.0 & 5.7116 & 0.603 \\
\hline 11 & 50 & 33.00 & 267.50 & 157.6 & 6.1785 & 0.520 \\
\hline 12 & 50 & 36.00 & 241.25 & 122.8 & 6.5055 & 0.442 \\
\hline 13 & 50 & 35.00 & 253.75 & 101.7 & 6.7603 & 0.356 \\
\hline 14 & 50 & 28.00 & 227.50 & 98.9 & 6.9713 & 0.277 \\
\hline 15 & 50 & 22.00 & 201.25 & 93.2 & 7.1765 & 0.205 \\
\hline 16 & 50 & 21.50 & 141.25 & 71.2 & 7.3699 & 0.153 \\
\hline 17 & 50 & 26.75 & 172.50 & 32.9 & 7.5176 & 0.088 \\
\hline 18 & 50 & 10.37 & 102.50 & 52.1 & 7.5859 & 0.054 \\
\hline 19 & 50 & 0 & 45.50 & & 7.6940 & 0.036 \\
\hline
\end{tabular}

ghezza del golfo di $1 \mathrm{~km}$ e di una profondità media di circa 6 metri. Essa ha condotto, tenendo conto della correzione di bocca valutata in 1,15 , ad un periodo per l'uninodale di $10^{\mathrm{m}}$ circa.

Il problema nel caso specifico doveva quindi essere affrontato sotto un aspetto più completo, non limitandoci alla considerazione dell'acqua ritenuta come fluido ideale, cosa questa che riesce pin che sufficiente nel caso di grandi bacini di considerevole profondità. 


\section{II. - a) L'AZIONE DELLA VISCOSITẢ SULLE OSCILLAZIONI LIBERE NEL PORTO DI CivitaveCCHA.}

I calcoli eseguiti per la determinazione dei periodi predominanti nelle oscillazioni secondarie, registrate nel porto di Civitavechia, hanno condotto, come si è visto, a risultati che si discostano per difetto da quelli osservati.

Non vi è dubbio che l'allungamento del periodo è in stretta relazione con la viscosità dell'acqua marina.

Sui movimenti fluidi a grande raggio, come i movimenti oceanici o atmosferici, la viscosità non influisce in modo decisivo, perchè quei movimenti provengono da condizioni termodinamiche. Così pure nei movimenti liberi di masse liquide profonde, risulta inapprezzabile l'azione della viscosità. Non così nel caso di moti interessanti masse liquide poco estese e poco profonde: le equazioni di movimento, dedotte nell'ipotesi di moti rigorosamente laminari, non rispecchiano, in questi casi, che in modo soltanto approssimativo l'andamento del fenomeno.

In tali condizioni, va tenuto conto dell'attrito (interno ed esterno), che non è certamente nullo in nessun fluido. La valutazione esatta degli effetti della viscosità urta contro difficoltà talvolta notevoli. Le equazioni dell'idrodinamica con i termini di viscosità si presentano più complicate che nel caso di fluidi ideali. Inoltre, nel miscuglio di masse animate da moti diversi, i coefficienti di viscosità che intervengono non sono più delle costanti, ma delle funzioni dello stato di turbolenza, e quindi, in generale, delle coordinate e del tempo.

Il problema si presenta quindi complesso. Generalmente, per studiare le azioni dell'attrito, si considera un movimento fluido, nel quale si hanno scorrimenti per piani orizzontali gli uni sugli altri. Si suppone inoltre costante la velocità, nello spazio e nel tempo, in ogni piano orizzontale $\left(^{4}\right)$.

In oceanografia dinamica, l'attrito sul fondo è ritenuto proporzionale al quadrato della velocità, mentre il corrispondente fattore di proporzionalità dipende solo dalla natura del fondo. In pratica, può valere pure la definizione fisica dell'attrito esterno, che ritiene la forza di attrito sul fondo proporzionale alla velocità di slittamento del liquido.

Va infine osservato che, date le condizioni di spazio e di tempo in cui si verificano i fenomeni da noi presi in esame, la rotazione della erra non può avere su di essi effetti sensibili. 
b) Caso di mancanza di corrente sul fondo.

Siano $u$ la velocità orizzontale della corrente (secondo l'asse delle $x$ ), $\mu$ e $\varrho$ il coefficiente di viscosità e la densità dell'acqua, $g$ l'accelerazione di gravità e $\zeta$ l'innalzamento della superficie provocato da una causa perturbante (p. es. il vento). Sarà $v=\mu / \varrho$ il coefficiente di viscosità cinematica (" eddy viscosity").

Nel piano verticale $x z$, prendiamo $z$ positivamente verso il basso dalla superficie indisturbata; la variazione di quest'ultima sia espressa da $z=-\zeta$.

Nell'ipotesi di mancanza di corrente sul fondo, il movimento dell'acqua in un canale unidirezionale, nello stato stazionario, è espresso da $\left(^{5-6}\right)$

$$
\mu \frac{\partial^{2} u}{\partial z^{2}}-g \varrho \frac{\partial \zeta}{\partial x}=0
$$

con le condizioni

$$
-\mu \partial u / \partial z=T(T, \text { tensione tangenziale del vento })
$$

alla superficie $(z=-\zeta)$,

$$
u=0 \quad \text { sul fondo } \quad(z=h)
$$

e

$$
S=\int_{-\zeta}^{h} u d z=0
$$

essendo $S$ il flusso totale, normale alla costa.

Due successive integrazioni della [1] danno

$$
\begin{gathered}
\mu \frac{\partial u}{\partial z}=g \varrho \frac{\partial \zeta}{\partial x} z+A, \\
\mu u=\frac{g \circ}{2} \frac{\partial \zeta}{\partial x} z^{2}+A z+B,
\end{gathered}
$$

rispettivamente.

Dalla [5], ricordando la [2], si ha

$$
T=g \varrho \frac{d \zeta}{d x} \zeta-A,
$$

mentre dalla [6], per la condizione [4], consegue

$$
\mu u=\frac{g \varrho}{2} \frac{d}{d x} \frac{\zeta}{x}\left(z^{2}-h^{2}\right)+A(z-h) .
$$


Or'a

$$
\int_{-\zeta}^{h} u d z=-\frac{1}{2 \mu}\left\{g \varrho \frac{d \zeta}{d x} \frac{2 h+3 h^{2} \zeta-\zeta}{3}+A(h+\zeta)^{2}\right\} .
$$

Dalla [4] si trae

$$
g \varrho \frac{d \zeta}{d x} \frac{2 h^{3}+3 h^{2} \zeta-\zeta^{3}}{3(h+\zeta)^{2}}+A=0
$$

Sostituendo nella [9] il valore di $A$ dato dalla [7], risulta

$$
\frac{d \zeta}{d x}=\frac{3}{2} \frac{T}{g \varrho(h+\zeta)},
$$

che dà l'inclinazione della superficie sotto l'azione del vento.

c) Caso di slitTamento StL Fondo.

Se esiste slittamento sul fondo di velocità $u_{h}$, nel qual caso la condizione sul fondo - in luogo della [3] — è data dalla

$$
-|\mu \partial u / \partial z|_{h=z}=f^{\prime} \varrho u_{h}
$$

essendo $f^{\prime}$ il coefficiente di attrito sul fondo, allora il primo integrale $[5]$ diviene

$$
-f^{\prime} \varrho u_{h}=g \varrho \frac{d \zeta}{d x} h+A
$$

La corrente di fondo $u_{h}$ si può pure trarre dal secondo integrale [6]

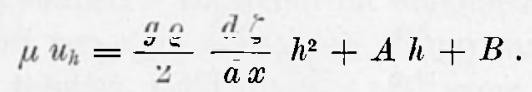

L'integrale da $-\zeta$ ad $h$ della $[137$ è:

$$
\frac{g \varrho}{2} \frac{d \zeta}{d x} \frac{h^{3}+\zeta^{3}}{3}+A \frac{h^{2}-\zeta^{2}}{2}+B(h+\zeta) ;
$$

per l'equazione di discontinuità [4] si ha pertanto

$$
\frac{g \varrho}{2} \frac{d \zeta}{d x} \frac{h^{2}-h \zeta-\zeta^{2}}{3}+A \frac{h-\zeta}{2}+B=0 \text {. }
$$

Le formule [7], [12], [13] e [14] permettono di determinare le quattro incognite $A, B, u_{h}, d \zeta / d x=\gamma$. Specialmente in mancanza di at- 
trito sul fondo $\left(f^{\prime}=0\right)$, l'inclinazione della superficie può trarsi da [7] e [12] soltanto, cioè

$$
\gamma=\frac{d \zeta}{d x}=\frac{T}{g o(h+\zeta)}
$$

Quando $f^{\prime} \neq 0$, come generalmente avviene, si può procedere nel seguente modo.

Dalle [7] e [12], per somma, si ha

$$
-f^{\prime} \underline{u_{h}}=g \underline{\rho} \gamma(h+\zeta)-T
$$

e, dalle [7] e [13],

$$
\mu u_{h}=g \varrho \gamma h\left(\frac{h}{2}+\zeta\right)-T h+B .
$$

Per cui, sostituendo nella [16] il valore di $u_{h}$ tratto dalla [15], si deduce

$$
\text { go } \gamma\left[\frac{\mu}{\check{I} \varrho}(h+\zeta)+h\left(\frac{h}{2}+\zeta\right)\right]-T\left(\frac{\mu}{f^{\prime} \varrho}+h\right)+B=0 .
$$

Dalle [7] e [14] si ha ancora

$$
g \circ \gamma\left[\frac{h^{2}-h \zeta+\zeta^{2}}{3}+(h-\zeta) \zeta\right]-\frac{T}{2}(h-\zeta)+B=0 .
$$

Combinando quest'ultima con la [17], consegue

$$
g \varrho \gamma(h+\zeta)\left[1+\frac{f^{\prime} \underline{o}(h+\zeta)}{3 \mu}\right]=T\left[1+\frac{f^{\prime} \varrho(h+\zeta)}{2 \mu}\right] ;
$$

da cui, posto

$$
N=\frac{1+\frac{f^{\prime} \varrho(h+\zeta)}{2 \mu}}{1+\frac{f^{\prime} \varrho(h+\zeta)}{3 \mu}},
$$

si ha

$$
\gamma=\frac{d \zeta}{d x}=\frac{N T}{g \varrho(h+\zeta)} .
$$

Si ha pertanto una formula analoga alla [10], corrispondente al caso in cui l'attrito sul fondo vale $f^{\prime} \varrho u_{h}$. Del tutto simile sarebbe la conclusione per un attrito sul fondo espresso da $f^{\prime} o u^{2}{ }_{h}$.

Va qui notato che il coefficiente $N$, dato dalla [18], si riduce a 1 se $f^{\prime}=0$ (assenza di attrito sul fondo) e a $3 / 2$ se $f^{\prime}=\infty$ (assenza di 
corrente sul fondo); e che, per valori finiti di $f^{\prime}$ e di $\mu$, il valore di $N$ per mare di piccola profondità $(h \sim 0$ ) tende a quello spettante ad assenza di attrito sul fondo, e per mare molto profondo a quello corrispondente ad assenza di corrente sul fondo.

Va ancora osservato che, poichè nella [18] $\zeta$ appare sia nel numeratore che nel denominatore in maniera similare, esso può essere omesso in pratica, il suo effetto riuscendo comparativamente lieve, laddove in [19] può occasionalmente assumere grande importanza.

III. - CAMBIAMENTo di STATO IN UN CANALE CON ATtRITo SUL Fondo FINITO; AZIONE COSTANTE SOPRA UN LAGO O UN MARE CHIUSO.

Sia $T$ un vento costante - o $\gamma_{0}$ un gradiente barometrico (misurato in colonna d'acqua) - , iniziante ad agire bruscamente su tutta l'acqua inizialmente in quiete, e venga provocata una variazione di livello (inclinazione) $\gamma=-\partial \zeta / \partial x$. L'equazione del moto sarà allora rappresentata da

$$
\begin{cases}\frac{\partial u}{\partial \imath}=\nu \frac{\partial^{2} u}{\partial z^{2}}+g \gamma & \text { per il vento } \nu=\frac{\mu}{\varrho} \\ \frac{\partial u}{\partial t}=v \frac{\partial^{2} u}{\partial z^{2}}+g\left(\gamma+\gamma_{0}\right) & \text { per il gradiente barom., }\end{cases}
$$

con le condizioni

$$
\begin{aligned}
& \text { alla superficie }(z=0)-\mu \partial u / \partial z=T \text { per azione del vento } \\
& -\mu \partial u / \partial z=0 \text { " } \begin{aligned}
-\mu \text { barometrica }
\end{aligned} \\
& \text { sul fondo }(z=h) \quad-\mu \partial u / \partial z=f^{\prime} \varrho u_{h} \\
& \text { inizialmente }(t=0) \quad u=\gamma=0
\end{aligned}
$$

e l'equazione di continuità

$$
\begin{gathered}
\frac{\partial \zeta}{\partial t}=-\frac{\partial S}{\partial x} \text { o } \frac{\partial \gamma}{\partial t}=\frac{\partial^{2} S}{\partial x^{2}} \\
\text { dove } S=\left.\right|_{0} ^{h} u d z .
\end{gathered}
$$

Un'espressione di $u$ che soddisfa le equazioni da [20] a [24] è la seguente ( $\left.{ }^{7}\right)$

$u=F \Sigma A_{n} \cos \beta_{n} z\left(1-e^{-\nu \beta_{n}^{2} t}\right)+\Sigma B_{n} \cos \beta_{n} z \int_{0}^{1} \gamma(\tau) e^{-\nu \beta_{n}{ }^{2}(t-\tau)} d \tau$ 
dove

$$
\beta_{n} \operatorname{tang} \beta_{n} h=f^{\prime} \stackrel{\varrho}{\mu}-\equiv
$$

$F=T$ per azione del vento

$F=\gamma_{0}$ per gradiente barometrico $\}$,

$A_{n}=\frac{2\left(\beta_{n}^{2}+k^{2}\right)}{h\left(\beta_{n}^{2}+k^{2}\right)+k} \cdot \frac{1}{\mu \beta_{n}^{2}} \quad$ per vento

$A_{n}=\frac{2\left(\beta_{n}^{2}+k^{2}\right)}{h\left(\beta_{10}^{2}+k^{2}\right)+k} \cdot \frac{g \sin \beta_{n} h}{\nu \hat{\beta}_{n}^{2}}$ per gradiente barometr.; |

$B_{n}-g \frac{2\left(\beta_{n}^{2}+k^{2}\right)}{h\left(\beta_{n}^{2}+k^{2}\right)+k} \cdot \frac{\sin \beta_{n} h}{\beta_{n}}$

Una verifica può essere eseguita per diretta sostituzione nelle equazioni da [20] a [24].

Ora, il flusso totale $\mathbb{S}$ diviene

$S=\int_{0}^{h} u d z=F \sum A_{n}-\frac{\sin \beta_{n} h}{\beta_{n}}\left(1-e^{-\nu \beta_{n}^{2} t}\right)+\Sigma B_{n} \frac{\sin \beta_{n} h}{\hat{\rho}_{n}} \int_{0}^{t} \gamma(\tau) e^{-\nu \beta_{n}^{2}(t-\tau)} d \tau$

soltanto termini con $n=0$ del quale possono essere introdotti nell'equazione di continuità [24]. Perciò abbiamo

$$
\frac{\partial \gamma}{\partial t}=\frac{a^{2} F^{\prime}}{d x^{2}} \frac{A_{0} \sin \beta_{0} h}{\beta_{0}}\left(1-e^{-\nu \beta_{0}^{2} t}\right)+B_{0} \frac{\sin \beta_{0} h}{\beta_{0}} \int_{0}^{t} \frac{\partial^{2} \gamma(\tau)}{\partial x^{2}} e^{-v \beta_{0}^{2}(t-\tau)} d \tau \text {. }
$$

Abbiamo ancora

$$
\frac{\partial^{2} \gamma}{\partial t^{2}}=\nu \beta_{0}^{2} \frac{d^{2} F}{d x^{2}} \frac{A_{0} \sin \beta_{0} h}{\beta_{0}} e^{-v \beta_{0}^{2} t}+B_{0} \frac{\sin \beta_{0} h}{\beta_{0}} \frac{\partial}{\partial t} \int_{0}^{t} \frac{\partial^{2} \gamma(\tau)}{\partial x^{2}} e^{-v \beta_{0}^{2}(t-\tau)} d \tau
$$

ora, per la regola di derivazione sotto il segno di integrali contenenti un parametro, con estremi variabili, si ha

$$
\frac{\partial}{\partial t} \int_{0}^{t} \frac{\partial^{2} \gamma(\tau)}{\partial x^{2}} e^{-v / \beta_{0}^{2}(t-\tau)} d \tau=-\int_{0}^{t} \frac{\partial^{2} \gamma(\tau)}{\partial x^{2}} \nu \beta_{0}^{2} e^{-\nu \beta_{0}(t-\tau)} d \tau+\frac{\partial^{2} \gamma(t)}{\partial x^{2}}
$$


Possiamo quindi scrivere

$$
\begin{gathered}
\frac{\partial^{2} \gamma}{\partial t^{2}}=-v \beta_{0}^{2}\left\{\frac{d^{2} F}{d x^{2}} \frac{A_{0} \sin \beta_{0} h}{\beta_{0}}\left(1-e^{-\nu \beta^{2}+}\right)+\left.B_{0} \frac{\sin \beta_{0} h}{\beta_{0}}\right|_{0} ^{t} \frac{\partial^{2} \gamma(\tau)}{\partial x^{2}} e^{-v \beta_{0}^{2}(t-\tau)} d \tau\right\}+ \\
+B_{0} \frac{\sin \beta_{0} h}{\beta_{0}} \frac{\partial^{2} \gamma}{\partial x^{2}}+\frac{d^{2} F}{d x^{2}} \frac{A_{0} \sin \beta_{0} h}{\beta_{0}} v \beta_{0}{ }^{2}
\end{gathered}
$$

Per la [31], si ha

$$
\frac{\partial^{2} \gamma}{\partial t^{2}}+v \beta_{0}{ }^{2} \frac{\partial \gamma}{\partial t}-R_{s} \frac{\sin \beta_{0} h}{\beta_{0}} \frac{\partial^{2} \gamma}{\partial x^{2}}=\frac{d^{2} F}{d x^{2}} \frac{A_{0} \sin \beta_{0} h}{\beta_{0}} v \beta_{0}{ }^{2}
$$

Se il canale è di lunghezza $L$, possiamo scrivere

$$
\left.\begin{array}{l}
\gamma=\sum \gamma_{m} \sin \frac{m \pi}{L} x, \frac{\partial^{2} \gamma}{\partial x^{2}}=\Sigma \gamma_{m}^{\prime \prime} \frac{\sin m \pi}{L} x, \gamma_{m}^{\prime \prime}=-\left(\frac{m \pi}{I}\right)^{\prime 2} \gamma_{m}, \\
F=\Sigma F_{m} \sin \frac{m \pi}{L} x, \frac{\partial^{2} F}{\partial x^{2}}=\sum F_{m}^{\prime \prime} \frac{\sin m \pi}{L} x, F_{m}^{\prime \prime}=-\left(\frac{m \pi}{L}\right)^{2} F_{m} .
\end{array}\right\}
$$

La [32] pertanto diviene

$\frac{d^{2} \gamma_{m}}{d t^{2}}+\nu \hat{\rho}_{0}{ }^{2} \frac{d \gamma_{m}}{d t}+D_{0} \frac{\sin \beta_{0} h}{\beta_{0}}\left(\frac{m \pi}{L}\right)^{2} \gamma_{m}=-A_{0} v \hat{\rho}_{0} \sin \beta_{0}{ }_{k}\left(\frac{m \pi}{L}\right)^{2} F_{m}$.

Posto

$$
\sigma_{m}=\sqrt{\Gamma_{0} \frac{\sin \beta_{0} \lambda}{\beta_{0}}\left(m \frac{\pi}{L}\right)^{2}-\left(\frac{\nu \beta_{0}^{-}}{2}\right)^{2}}
$$

nell'equazione differenziale a coefficienti costanti [3t], l'equazione omogenea ha l'integrale

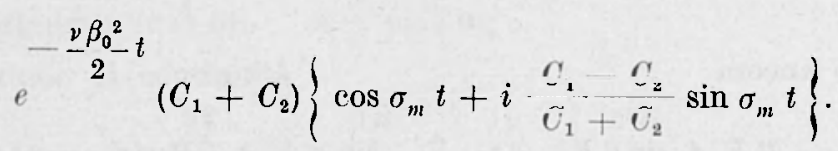

Un integrale particolare della [3t] è

$$
-\frac{A_{0}}{B_{0}} v \beta_{0}^{2} F_{m}
$$

L'integrale generale della [3t] è pertanto $\gamma_{m}(t)=e^{-\frac{1}{2} \nu \beta_{2}^{2} t}\left(C_{1}+C_{2}\right)\left\{\cos \sigma_{m} t+i \frac{C_{1}}{U_{1}+C_{2}} \sin \sigma_{m} t\right\}-\frac{A_{n}}{\bar{B}_{0}} \nu \beta^{2} F_{m}$. 
Al tempo $t=0, \gamma=\gamma_{m}=0$, pertanto

$$
C_{1}+C_{2}=\frac{A_{0}}{B_{0}} v \beta_{0}^{2} F_{m}
$$

E inoltre, per $t=0, \frac{d \gamma_{m}}{\vec{u} t}-=0$; ne consegue

$$
i \frac{C_{1}-C_{2}}{C_{1}+C_{2}}-\frac{\nu \beta_{0}^{2}}{2 \sigma_{m}^{2}}
$$

Pertanto, l'integrale generale della [34] diviene

$$
\begin{aligned}
& \gamma_{m}=-\frac{A_{n}}{B_{0}} \nu \beta_{0}^{2} F_{n}\left[1-e^{-1 / 2 v \beta_{0}^{2} t_{1}}\left\{\cos \sigma_{m} t+\frac{\nu_{1}^{\prime-v}{ }^{2}}{2 \sigma_{m}} \sin \sigma_{m} t\right\}\right], \\
& \gamma_{m}=-\frac{A_{0}}{B_{0}} \nu \beta_{0}^{2} F_{n}\left[1-\frac{\sqrt{4 \sigma_{m}^{2}+\left(\nu \beta_{0}^{2}\right)^{2}}}{2 \sigma_{m}} e^{-1 / 2 v \beta_{0}^{2} t} \cos \left(\sigma_{m} t-\varepsilon\right)\right],
\end{aligned}
$$

dove

$$
\operatorname{tang} \varepsilon=\frac{\nu \beta_{0}}{2 \sigma_{m}}
$$

La [35] mostra che l'andamento della perturbazione sarà oscillatorio, oppure no, a seconda che è

$$
\frac{4 B_{0} \sin \beta_{0} h}{\nu^{2} \beta_{0}{ }^{5}}\left(\frac{m \pi}{L}\right)^{2}>1 \text { oppure }<1 .
$$

Il fattore di smorzamento è evidentemente $e^{-1 / 2 v \beta_{0}^{2}}$

Y. - ApPlicazione alla Determinazione Della viscosità DeLL'ACQUA DEL gOlfo DI Civitavecchia.

Quello di Civitavecchia è un golfo con i fondali generalmente molto bassi (fig. 2). Le oscillazioni libere delle sue acque devono quindi risentire dell'effetto della viscosità. È quanto ci siamo proposti di determinare con il calcolo. La formula

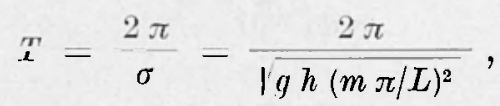

nota come formula di Merian, dà il periodo nel caso di fluido ideale.

Dall'elaborazione di lunghissime serie di registrazioni di oscillazioni uninodali, ottenute negli ultimi anni dal mareografo funzionante nel 

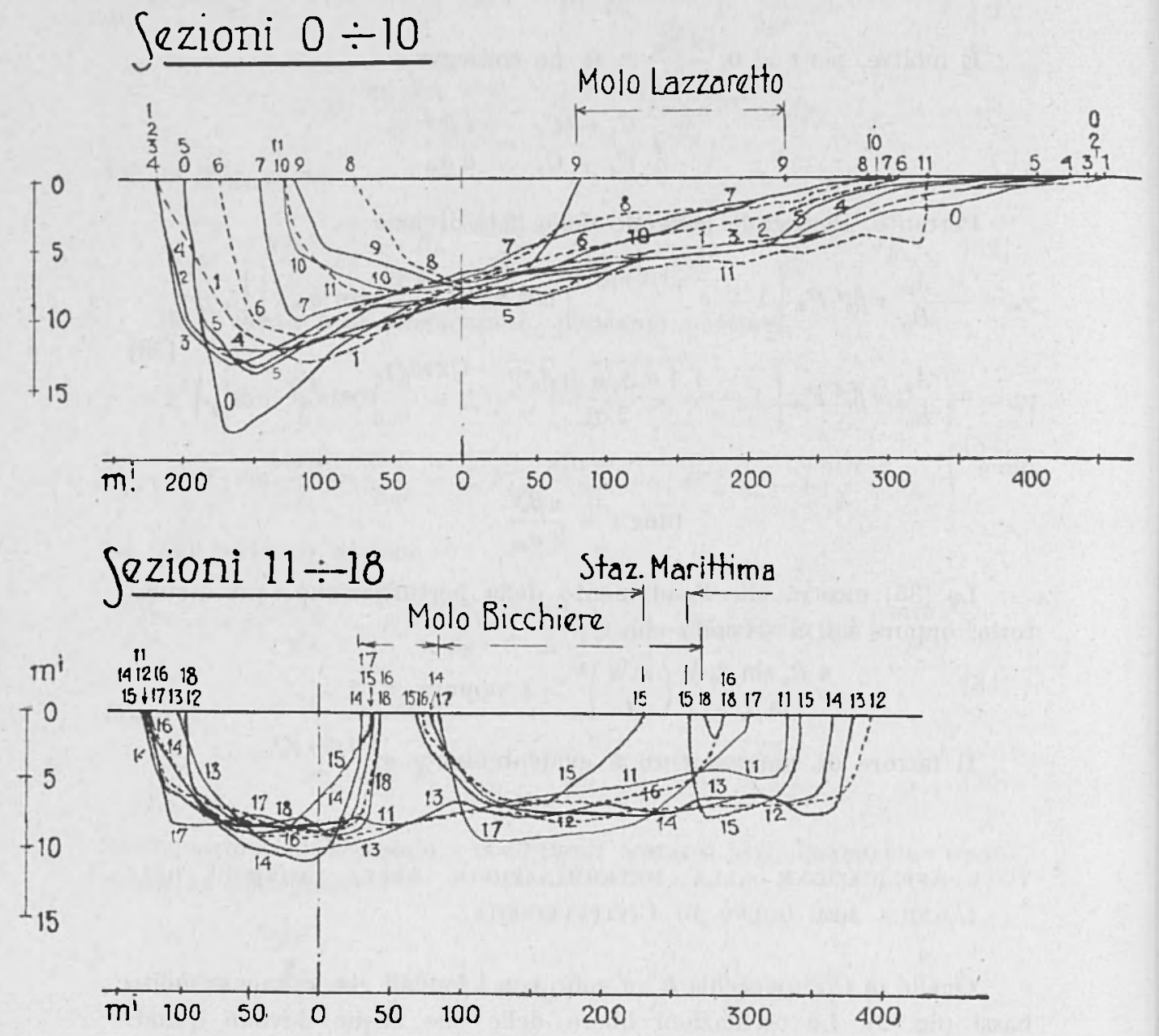

Fig. 2 
porto di Civitavecchia, si è ottenuto per il rapporto di smorzamento. relativo a mezzo periodo (e valutato al cessare della causa perturbante) il valore medio

$$
0,45
$$

Ciò consente di ottenere per l'indice di smorzamento per mezzo periodo dell'oscillazione di $11_{\mathrm{m}}$ circa, il valore

$$
\frac{1}{2} v \beta_{0}^{2}=2,42.10^{-3} \text { sec. }^{-1}
$$

A causa della viscosità, a $\sigma$ spetta l'espressione data dalla [35]. Quest'ultima, pertanto, può ritenersi corrispondere ai valori forniti dall'osservazione; indicando con $\sigma_{v}$ il valore relativo all'uninodale nel caso del fluido reale e con $\sigma_{o}$ quello spettante al fluido ideale, dalle [35] e [37] si trae (per $m=1$ )

$$
\begin{aligned}
& \sigma_{0}{ }^{2}=g h(\pi / L)^{2} \\
& \sigma_{v}{ }^{2}=B_{0} \frac{\sin \beta_{0} h}{\beta_{0}}\left(\frac{\pi}{L}\right)^{2}-\left(\frac{1}{2} v \beta_{0}\right)^{2},
\end{aligned}
$$

da cui

$$
\frac{B_{0}}{g} \frac{\sin \beta_{0} h}{\beta_{0} h}=\frac{\sigma_{\nu}^{2}+\left(\frac{1}{2} v \beta_{0}\right)^{2}}{\sigma_{0}^{2}}
$$

La determinazione di $\sigma_{0}$ presuppone la conoscenza del periodo. Si puó usare la [37], tenendo conto che qui trattasi di una baia. Pertanto, sarà $L$ uguale al doppio della lunghezza di quest'ultima, che è di m 1000 . Inoltre, la profondità media della baia è di metri 6 ; quindi, $h=\mathrm{m} 6$. Allora, dalla [37], fatto $m=1$ (uninodale), si trae

$$
T_{0}=8^{\mathrm{m}}, 6
$$

Dobbiamo poi tener conto della correzione di bocca $\left({ }^{8}\right)$.

Nel caso in esame, essa è risultata pari a 1,15 . Il periodo corretto, nel caso di fluido ideale, è quindi

$$
T=10^{\mathrm{m}} \text { circa }
$$

Ne consegue

$$
\sigma_{0}=1,05 \cdot 10^{-2}
$$

Il valore osservato per il periodo dell'oscillazione libera uninodale è

$$
T=10^{\mathrm{m}}, 8 .
$$


Scegliendo, in cifra tonda, $11^{\mathrm{m}}$, avremo

$$
\sigma_{0}=9,5 \cdot 10^{-3}
$$

Sostituendo nella [38], ne consegue

$$
\frac{B_{0}}{g} \frac{\sin \beta_{0} h}{\beta_{0} h}=C=\hat{0.07} \text {. }
$$

Ora, per $n=0$, dalla [29] - tenuto conto della [26] - si deduce

$$
\frac{B_{0}}{g} \frac{\sin \beta_{0} h}{\beta_{0} h}=\frac{2\left(1+t g^{2} \beta_{0} h\right)}{1+t g^{2} \beta_{0} h+\frac{\operatorname{tg} \beta_{0} h}{\beta_{0} h}}\left(\frac{\sin \beta_{0} h}{\beta_{0} h}\right)^{2} .
$$

Ricordando la [39] si ottiene

e quindi

$$
\left.\left(\frac{\sin \beta_{n} h}{\beta_{0} h}\right)^{2}=\frac{C}{2} \zeta_{i} \div \frac{\sin 2 \beta_{0} h}{2 \beta_{0} h}\right)
$$

$$
4 \sin ^{2} \beta_{0} h=2 C\left(\beta_{0} h\right)^{2}+C \beta_{0} h \sin 2 \beta_{0} h .
$$

Dando a $C$ il valore [39], si ha

$$
\beta_{0} h=1,44 \text {. }
$$

Poichè la profondità media è $h=6 \mathrm{~m}$, ne consegue

$$
\beta_{0}=0,0024 \mathrm{~cm}^{-1} \text {. }
$$

Dal valore dell'indice di smorzamento $12 v \beta_{0}{ }^{2}$, abbiamo

$$
v=\frac{2 \times 2,42 \times 10^{-3}}{5,76 \times 10^{-6}}=840 \quad \text { c.g.s. }
$$

Abbiamo già notato che, per piccoli valori della profondità, il valore di $N$ è prossimo all'unità. Tale è il caso in esame; e poichè il valore di $f h / v$ è molto piccolo, tenuto conto della [26] per $n=0$, si trae

$$
\begin{aligned}
& f^{\prime} \cong 2 h\left(\begin{array}{ll}
1 & v \beta_{1}^{2} \\
2
\end{array}\right) \\
& f^{\prime}=2,9 \quad \text { c.g.s. }
\end{aligned}
$$

Questo è però soltanto un valore approssimato per $f^{\prime}$, che può essere tratto direttamente dalla [26] - per $n-0-$, una volta noti $\beta_{o}, \beta_{o} h, \nu$. Con $\mathrm{i}$ valori già calcolati $-\beta_{0} h=1,44 ; \nu=840$ - si ottiene infatti dalla $[26]$

$$
f^{\prime}=15,3 \text { c.g.s. }
$$


Ciò consente anzi di determinare, con maggior approssimazione, quale valore compete realmente ad $N$. Infatti, dalla [18] per $\zeta=0$, per $v=840, f^{\prime}=15,3$ e $h=600 \mathrm{~cm}$; consegue

$$
N=1,39\left({ }^{*}\right) \text {. }
$$

Abbiamo cosi ottenuto i valori del coefficiente di attrito sul fondo (assunto linearmente proporzionale alla velocità di slittamento) $f^{\prime} \mathrm{e}$ del coefficiente di viscosità cinematica (" eddy viscosity ") $\nu$, associati alle normali oscillazioni libere uninodali del golfo di Civitavecchia. I loro valori variano - a parità di altre condizioni - con l'ampiezza e crescono al crescere di quest'ultima. Nel caso considerato - attesa la piccola profondità - la corrente sul fondo non è molto minore di quella in superficie. I calcoli furono condotti su questa ipotesi.

V. - Restava da verificare, per via indiretta, il valore della viscosità cinematica, ottenuto nel paragrafo precedente.

Qualora questo fosse stato attendibile, sostituiti nella [35] — fatto $m=1$, per l'uninodale - i valori numerici dei termini $\frac{B_{o} \sin \beta_{o} h}{\hat{\rho}_{o} \bar{h}}$ e $1 / 2 v \beta_{o}{ }^{2}$, ottenuti nelle ipotesi fatte, si sarebbe dovuto ottenere per $\sigma_{1}$ - e quindi per $T_{1}-$ un valore prossimo a quello conseguente alle osservazioni.

Fatti i calcoli si è infatti ottenuto

$$
T_{1}=\frac{2 \pi}{\sigma_{1}}=9^{\mathrm{m}}, 4
$$

che, moltiplicato per il fattore di correzione di bocca 1,15 , dà

$$
T_{1}=10^{\mathrm{m}}, 8 \text {, }
$$

che coincide effettivamente con la media dei valori osservati per l'uninodale del golfo.

VI. - Circa le oscillazioni libere osservate nel porto di Civitarecchia, va detto che predomina fra esse, la uninodale. Non abbiamo ancora avuto modo di elaborare un cospicuo numero di mareogrammi, ma da quelli già sottoposti a spoglio risulta chiaro che l'uninodale $\grave{e}$ sempre presente e insorge di continuo, con estrema facilità. Fra le non molte

(*) Nel caso analogo, considerato da $T$. Nomitsu, anziché $N-1$, come presuppone litutore, il calcolo fornisce $N-1,36$. 
osservazioni esaminate, abbiamo notato spesso ampiezze ragguardevoli, dell'ordine di 20 e più centimetri. Sovente, sovrastano la stessa marea.

E nostro proposito iniziare - non appena possibile - una serie sistematica di osservazioni, con apparecchi più adatti a questo fine.

A scopo sperimentale, furono eseguite alcune registrazioni presso la Capitaneria di Porto, in due giorni di perfetta calma del mare. La sessa uninodale - sia pure di piccola ampiezza - è stata sempre presente. Ci limitiamo a riportare l'esempio dato dalle figg. $3,4,5,6,7,8,9,10$.

In essa, oltre all'uninodale, si vede - sovrapposta a quest'ultima, a partire dalle ore 18,35 - una serie di altre oscillazioni libere, di minor periodo. La più ampia, ha un periodo di $3^{\mathrm{m}}$ circa, poco più. Più che di una oscillazione multinodale, interessante l'intera baia, riteniamo trattarsi di una sessa uninodale di una parte del porto, e precisamente di quella che ha la sua imboceatura fra il molo Bicchiere e il molo Lazzaretto. Fatti i calcoli approssimativi, tale parte del porto infatti, risulta avere - tenuto conto della correzione di bocea e per una profondità media, di $6 \mathrm{~m}$ circa - un'oscillazione libera uninodale dell'ordine di $3^{\mathrm{m}}, 25$ circa, in buon accordo quindi con quella osservata.

Sia di queste oscillazioni, sia sopra tutto della causa prima che provoca l'insorgere nel porto dell'uninodale dell'intero golfo, ci proponiamo di interessarci nel proseguimento della ricerca.

\section{RIASSUNTO}

Scopo di questo lavoro era lo studio delle oscillazioni libere del porto di Civitavecchia.

L'applicazione delle ordinarie teorie termodinamiche, condussero però a valori troppo diversi - per difetto - da quelli osservati. L'acqua non poteva essere quindi ritenuta - nel caso in esame - come fluido ideale. Data la piccola profondità media del porto (6 metri ca.), e la sua relativa estensione, la viscosità doveva influire sull'allungamento del periodo.

Esaminato questo aspetto del problema, l'applicazione di una teoria sull'effetto della viscosita cinematica, ha portato ad ottimi risultati. Per l'uninodale del golfo si è ottenuto un valore teorico $\left(T=10^{m}, 8\right)$, coincidente con la media di quelli osservati. L'allungamento del valore del periodo, quale si avrebbe avuto nel caso di fluido ideale, è risultato corrispondente all'azione della viscosità dinamica, per la quale si è ottenuto $v=840 \mathrm{c.g.s}$. 


\section{$A B S T R A C T$}

The aim of the present work is the study of free oscillations in the Port of Civitavecchia.

The application of ordinary thermodynamic theories has yielded, however, values too shorter than those observed. Thus, in this particular case, water could not be considered an ideal fluid. In view of the modest depth of the port (about 141/2 feet) and its comparatively considerable extent, it must be supposed that viscosity influenced the increase of the period.

After having considered this aspect of the problem, a theory concerning the effect of kinematic viscosity was applied yielding excellent results. A theoretical value $\left(T=10^{\mathrm{m}}, 8\right)$ coinciding with the average observed was obtained for the uninodal of the gulf. The lengthening of the period which would take place in the case of an ideal fluid has turned out corresponding to the action of dynamic viscosity for which we obtained $\nu=810$ c.g.s.

\section{BIBLIOGRAFIA}

(1) Calor, P., Sesse dell'alto Adriatico, con particolare riguardo al golfo di Trieste, Memoria CCXLVII del R. Comitat. Talass. It., Venezia 1938.

(2) Caloi, P. - Marcelli, L., Oscillazioni libere del golfo di Napoli, "Annali di Geofisica ", II, (1949).

( $\left.{ }^{3}\right)$ Calor, P., Le sesse del Lago di Garda, Parte II, "Annali di Geofisica ", I, (1948).

(4) Bjerknes, V. - Bjerknes, J. - Solberg, H., - Bergeron, T., Hydrodynamique physique, I, 187.

$\left(^{5}\right)$ Nomitsif, T. - TAKEgAMI, T., Coast effect upon the Ocean Current and the Sea Level. I. Steady State. "Memoirs College Science Kyoto Imp. Univ. ", XVII, 3, (1934).

${ }^{(6)}$ Nonitsu, T., Coast effect upon the Ocean Current and the Sea Level. II. Changing State. "Memoirs College Science Kyoto Imp. Univ.", XVII 5, (1934).

(7) - A Theory of Tunamis and Seiches... "Memoirs College Science, Kyoto Univ. ", XVIII, 4, (1935).

(8) Caloi, P., Spadea, M. C., Le sesse del Lago di Garda. Parte IV. "Annali di Geofisica ", VI, (1953). 


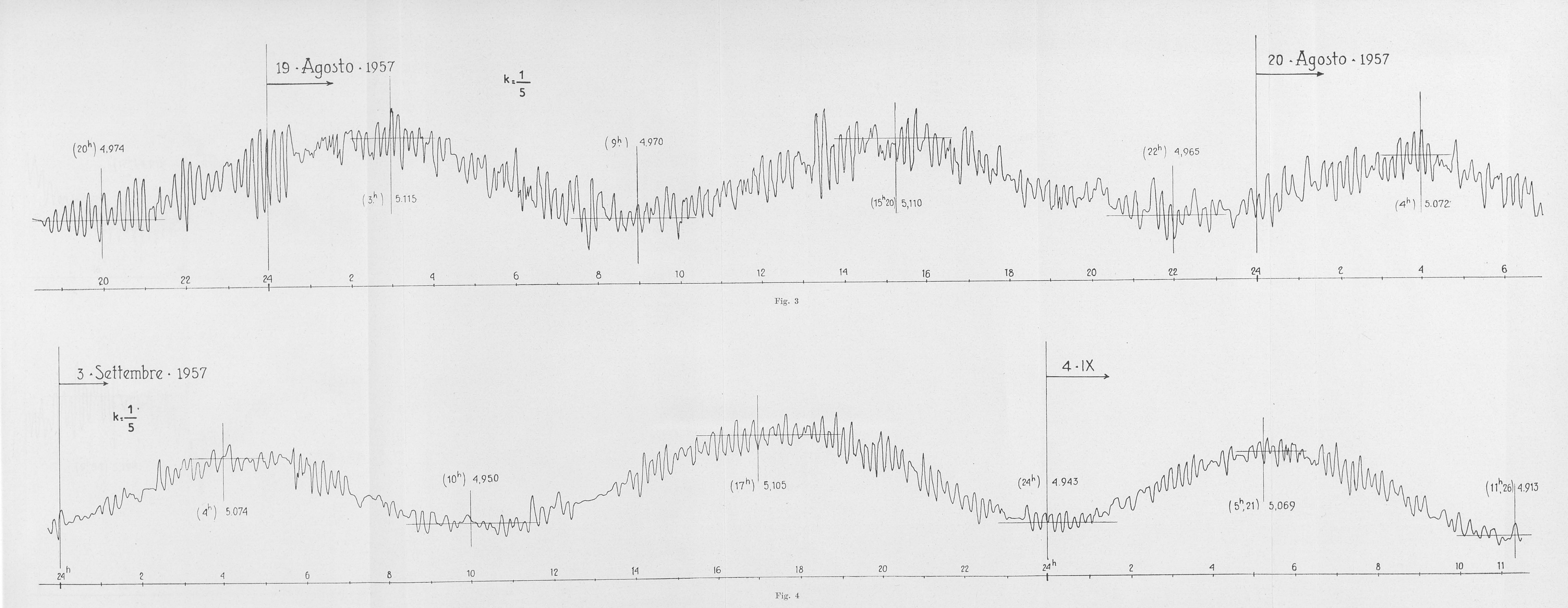




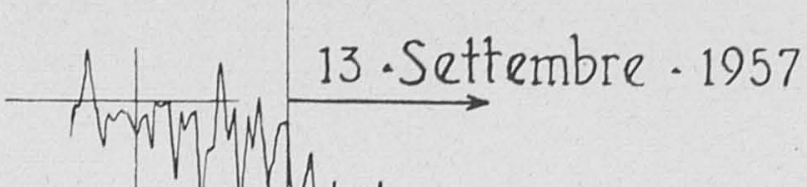
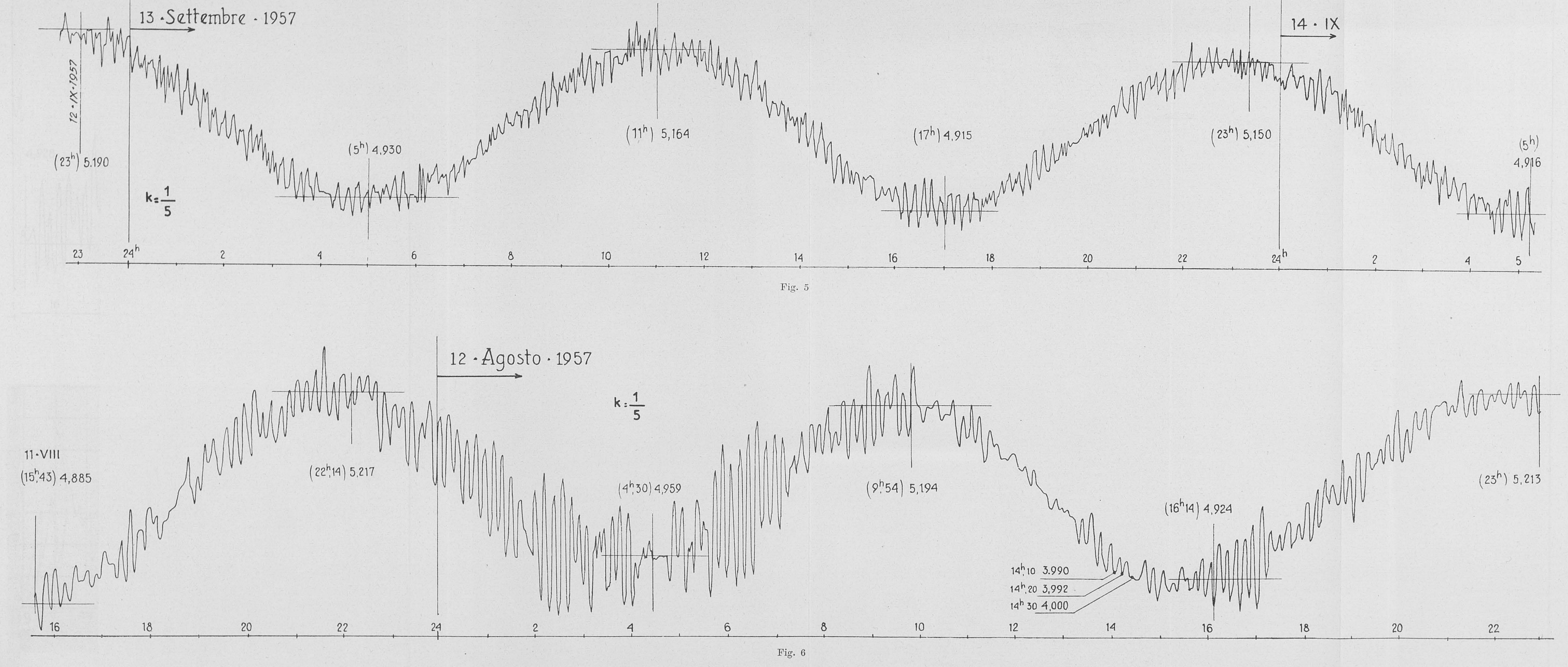

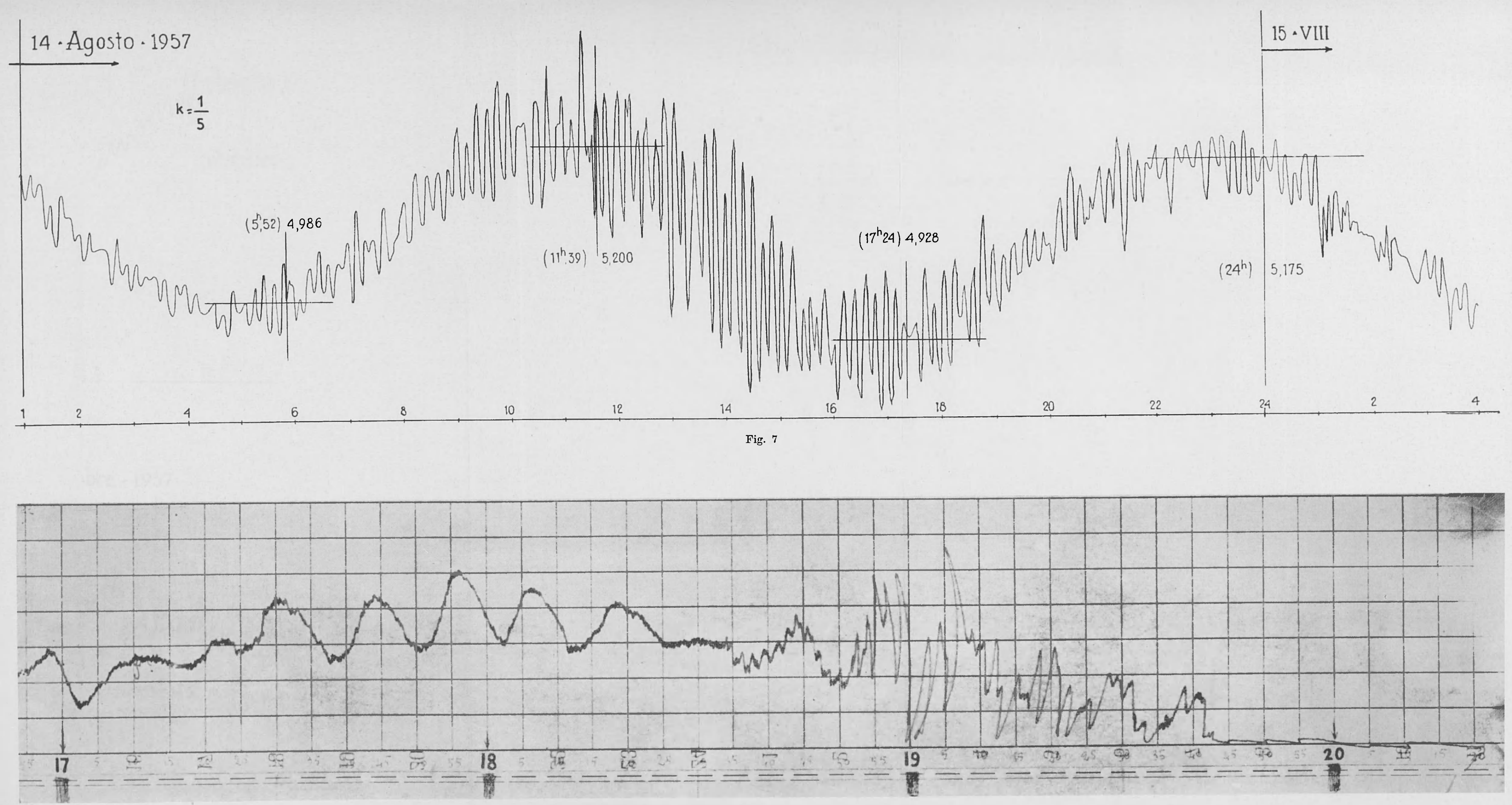

Fig. 8 

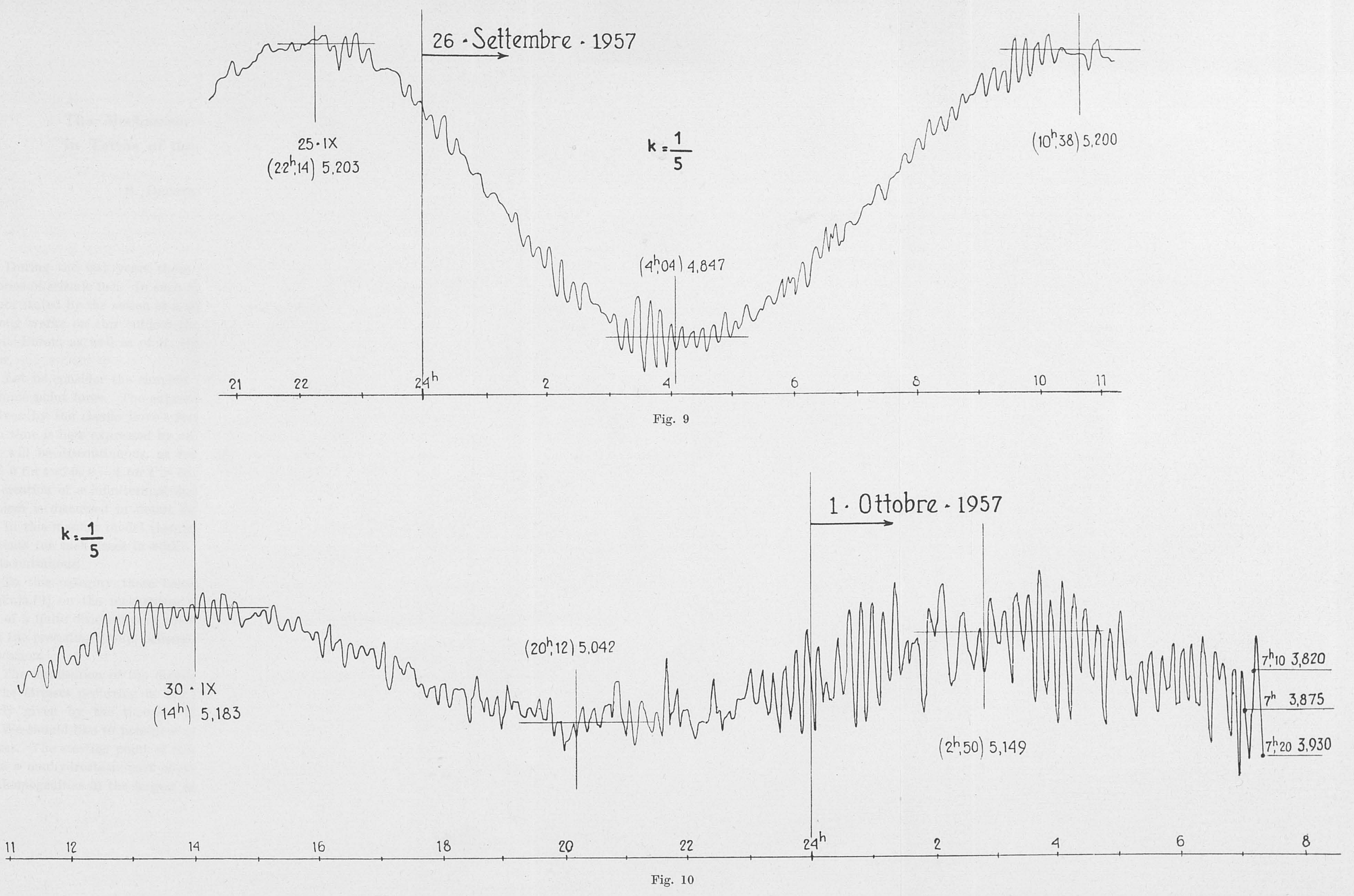